\title{
Reactivated Spatial Context Guides Episodic Recall
}

\author{
๑Nora A. Herweg, ${ }^{1}$ Ashwini D. Sharan, ${ }^{2}{ }^{\circledR}$ Michael R. Sperling, ${ }^{3}$ Armin Brandt, ${ }^{4}{ }^{\circledR A n d r e a s ~ S c h u l z e-B o n h a g e, ~}{ }^{4}$ \\ and ${ }^{-M i c h a e l ~ J . ~ K a h a n a ~}{ }^{1}$ \\ ${ }^{1}$ Computational Memory Laboratory, Department of Psychology, University of Pennsylvania, Philadelphia, Pennsylvania 19104, Departments of \\ ${ }^{2}$ Neurosurgery, ${ }^{3}$ Neurology, Thomas Jefferson University, Philadelphia, Pennsylvania 19107, and ${ }^{4}$ Epilepsy Center, University Medical Center, Freiburg \\ 79110, Germany
}

The medial temporal lobe (MTL) is known as the locus of spatial coding and episodic memory, but the interaction between these cognitive domains as well as the extent to which they rely on common neurophysiological mechanisms is poorly understood. Here, we use intracranial electroencephalography and a hybrid spatial-episodic memory task (29 subjects, 15 female) to determine how spatial information is dynamically reactivated in subregions of the human MTL and how this reactivation guides recall of episodic information. Our results implicate theta oscillations across the MTL as a common neurophysiological substrate for spatial coding in navigation and episodic recall. We further show that our index of retrieved spatial context is high in the hippocampus (HC) in an early time window preceding recall. Closer to recall, it decreases in the $\mathrm{HC}$ and increases in the parahippocampal gyrus. Finally, we demonstrate that hippocampal theta phase modulates parahippocampal gamma amplitude during retrieval of spatial context, suggesting a role for crossfrequency coupling in coding and transmitting retrieved spatial information.

Key words: episodic memory; hippocampus; intracranial EEG; MTL; reinstatement; theta

\section{Significance Statement}

By recording from the human medial temporal lobe (MTL) while subjects recall items experienced in a virtual environment, we establish a direct relation between the strength of theta activity during memory search and the extent to which memories are organized by their spatial locations. We thereby pinpoint a role for theta oscillations in accessing the "cognitive map" during episodic retrieval and further highlight the dynamic interplay of hippocampus and extrahippocampal MTL in representing retrieved spatial context. Our results provide an important step toward a unified theory of MTL function encompassing its role in spatial navigation and episodic memory.

\section{Introduction}

Spatiotemporal context provides a unique tag for each event we experience, and the similarities among these tags organize the contents of episodic memory. This organization can be inferred from the way people recall information. When remembering lists of words, people tend to successively recall words from neighbor-

Received July 11, 2019; revised Nov. 21, 2019; accepted Nov. 23, 2019.

Author contributions: N.A.H., A.D.S., M.R.S., A.S.-B., and M.J.K. designed research; N.A.H., A.D.S., and A.B. performed research; N.A.H. analyzed data; N.A.H. and M.J.K. wrote the paper.

This work was supported by National Institutes of Health Grant MH061975 to M.J.K. and by Deutsche Forschungsgemeinschaft Grant HE 8302/1-1 to N.A.H. We thank Christoph Weidemann for help with data analyses; Paul Wanda, Alison Xu, Zeinab Helili, Katherine Hurley, Deb Levy, Logan 0'Sullivan, Ada Aka, and Allison Kadel for help with data acquisition and postprocessing; Jonathan Miller and Ansh Johri for their contributions to the task design; Corey Novich and Ansh Patel for programming the Unity-based experiment; and Joel Stein, Rick Gorniak, and Sandy Das for electrode localization support. We thank all patients and their families who selflessly volunteered their time to participate in this research.

The authors declare no competing financial interests.

Correspondence should be addressed to Nora A. Herweg at nherweg@sas.upenn.edu or Michael J. Kahana at kahana@psych.upenn.edu.

https://doi.org/10.1523/JNEUROSCl.1640-19.2019

Copyright $\odot 2020$ the authors ing list positions (Kahana, 1996; Healey et al., 2019). More recent research has shown that spatial context similarly guides recall transitions. During recall of items presented in a virtual environment, subjects successively recall items studied at proximate spatial locations (Miller et al., 2013a). These results suggest that temporal and spatial context are reactivated during recall and serve as a cue for associated items. They further establish a link between spatial coding and episodic memory, two domains that have been associated with the medial temporal lobe (MTL) in parallel lines of research.

During navigation, single cells in the hippocampus (HC) and entorhinal cortex (EC) represent an animal's location in space (O'Keefe, 1979; Ekstrom et al., 2003; Hafting et al., 2005; Jacobs et al., 2013), and hippocampal low-frequency oscillations accompany the firing of these cells (Vanderwolf, 1969; Buzsáki et al., 1983; O'Keefe and Recce, 1993; Ekstrom et al., 2005; Cornwell et al., 2008; Koenig et al., 2011; Aghajan et al., 2017; Bohbot et al., 2017; Bush et al., 2017; Miller et al., 2018). In humans, delta-theta power increases during successful navigation (Cornwell et al., 2008; Kaplan et al., 2012) and spatial memory encoding (Miller et 
al., 2018). Together, these results suggest that low-frequency oscillations orchestrate place and grid cell firing to support navigation (Herweg and Kahana, 2018).

In the episodic memory domain, the $\mathrm{HC}$ and surrounding parahippocampal gyrus (PHG) constitute the MTL memory system (Squire and Zola-Morgan, 1991), a system thought to form and retrieve event memories by associating arbitrary stimulus combinations. Despite the striking anatomical overlap of spatial coding and episodic memory, remarkably little is known about potential shared neurophysiological mechanisms. Buzsáki (2005) has proposed that theta oscillations support the formation of both spatial and episodic associations by organizing spike timing and associated plasticity, but evidence in favor of this idea is scarce. In fact, a widespread decrease in low-frequency power and increase in high-frequency power often accompanies successful episodic memory operations (Burke et al., 2014; Kragel et al., 2017; Weidemann et al., 2019; Solomon et al., 2019). More localized increases in temporal narrow-band theta oscillations during successful encoding (Lega et al., 2012) and retrieval (Burke et al., 2014; Weidemann et al., 2019) have also been observed and linked to recollection of context (Guderian and Düzel, 2005; Herweg et al., 2016).

Here, we use a spatial-episodic memory task and intracranial electroencephalography (EEG) to assess the role of low-frequency and high-frequency activity for spatial context retrieval in the MTL. On each trial, subjects delivered objects to a series of stores in a virtual town and subsequently recalled those objects (Fig. 1a,b). We hypothesized that retrieval of spatial context, in contrast to general biomarkers of successful memory, is accompanied by increased theta power. Next, we asked whether there is evidence for reactivation of spatial representations, and whether the timing of reactivation differs between $\mathrm{HC}$ and $\mathrm{PHG}$. Prior research has shown that place cells reinstate their activity during recall of words that were encoded in the cells' place fields (Miller et al., 2013 b), but the temporal dynamics of reinstatement in different MTL subregions are currently unknown. Finally, we asked how information is transferred between the HC and PHG. Theta oscillations have been implicated in inter-regional connectivity during successful memory (Watrous et al., 2013; Herweg et al., 2016; Solomon et al., 2017, 2019), and place-responsive cells are locked to both theta and gamma oscillations (Skaggs et al., 1996; Senior et al., 2008). Based on the idea that assemblies of neurons are organized by a theta-gamma code (Dragoi and Buzsáki, 2006; Lisman and Jensen, 2013), we explore theta-gamma coupling between $\mathrm{HC}$ and PHG during recall of spatial context.

\section{Materials and Methods}

Participants. Twenty-nine patients ( 15 female, 14 male; mean \pm SD age, $35 \pm 11$ years) with medication-resistant epilepsy who were undergoing clinical seizure monitoring at Thomas Jefferson University Hospital (Philadelphia, PA), the University Clinic Freiburg (Freiburg, Germany), and the Hospital of the University of Pennsylvania (Philadelphia, PA) participated in the study. The study protocol was approved by the Institutional Review Board at each hospital, and subjects gave written informed consent. Electrophysiological data were recorded from depth electrodes placed, according to clinical considerations, in the $\mathrm{HC}$ and/or surrounding PHG.

Experimental design. Subjects played the role of a courier in a hybrid spatial-episodic memory task, riding a bicycle and delivering parcels to stores located within a virtual town (consisting of roads; stores; taskirrelevant buildings; areas of grass; and props such as fences, streetlights, and trees; Fig. 1a,b). Each experimental session consisted of a series of delivery days (i.e., trials), during which subjects navigated to deliver objects and, at the end of each trial, recalled those objects. Subjects com- pleted slightly different versions of this paradigm, the details of which are described in the following paragraphs. These versions were programmed and displayed to subjects using the Panda Experiment Programming Library (Solway et al., 2013), which is a Python-based wrapper around the open-source game engine Panda3d (with 3D models created using Autodesk Maya) or the Unity Game Engine (Unity Technologies).

Before starting the first delivery day, subjects viewed a static or rotating rendering of each store in front of a black background. Each store had a unique storefront and a sign that distinguished it from task-irrelevant buildings. This "store familiarization" phase was followed by a "town familiarization" phase, in which subjects were instructed to navigate from store to store without delivering parcels (and recalling objects), visiting each store one to three times in pseudorandom order (each store was visited once, before the first store was visited the second time). Subjects were informed about their upcoming goal by on-screen instructions and navigated using the joystick or buttons on a game pad. Locationstore mappings in the town were fixed for seven subjects and random for 22 subjects (the layout was always fixed across experimental sessions, i.e., each subject experienced the same town layout across sessions). For a subset of the subjects, the town and store familiarization phases were not only part of the first but also part of all following sessions, with just one visit to each store before the first delivery day in each session. Furthermore, waypoints helped a subset of subjects navigate upon difficulties finding a store. Considering each intersection as a decision point, arrows pointing in the direction of the target store appeared on the street after three bad decisions (i.e., decisions that increased the distance to the target store).

Each delivery day trial consisted of a navigation phase and a free recall phase (and for some subjects, an additional cued recall phase following free recall, for which no data are reported here; Fig. 1a). For the navigation phase, 13 stores of the total number of 16 or 17 stores were chosen at random. To minimize any relationship between spatial and temporal proximity, the sequence of these 13 target stores was also selected at random. Across subjects, the average correlation of spatial and temporal proximity (calculated as the percentile ranking of the distances between each encoding location/time point and all other encoding locations/time points on the same list) was $r_{M}=-0.012\left(r_{\max }=0.31, r_{\min }=-0.17\right)$. Subjects navigated to these stores sequentially (including on-screen instructions and waypoints described earlier). Upon arrival at the first 12 stores, subjects were presented with an audio of a voice naming the object $(N=23$ subjects; variable duration on the order of 1-2 s) or an image of the object ( $N=6$ subjects; $5 \mathrm{~s})$ they just delivered. Objects were drawn with and without replacement for 15 and 14 subjects, respectively. If objects were repeated, they were repeated on different trials. Objects never repeated within one trial. For 23 subjects, objects were semantically related to their target store to aid recall performance. Object presentation was followed by the next on-screen navigation instruction (e.g. "Please find the hardware store"). Upon arrival at the final store, where no item was presented, the screen went black and subjects heard a beep. After the beep, they had $90 \mathrm{~s}$ to recall as many objects as they could remember in any order. Vocal responses were recorded and annotated offline. Subjects completed a variable number of delivery days per session (minimum, 2; maximum, 14; mean, 6). A final free recall phase followed the last delivery day within each session, for which no data are reported here.

Behavioral analyses of recall transitions. Behavioral data were analyzed using Python version 2.7 (Python Software Foundation). We assessed organization of recall sequences by retrieved spatial and temporal context with the same method: we assigned each recall transition the Euclidean (temporal) distance between the two encoding locations (time points). In the same way, we calculated the distance for all possible transitions that could have been made instead of each actual transition (i.e., the distance between the location/time point of the first item in each transition and all locations/time points at which objects were encoded on the very same list that had not yet been recalled in the very same recall phase). We then calculated a spatial (temporal) clustering score for each recall transition as 100 minus the percentile ranking of the spatial (temporal) distance assigned to the actual transition with respect to all possible transitions. The higher the average spatial (temporal) clustering score was across recall transitions, the more likely a subject was to transition between items that were encoded at proximate locations (time points). 
a
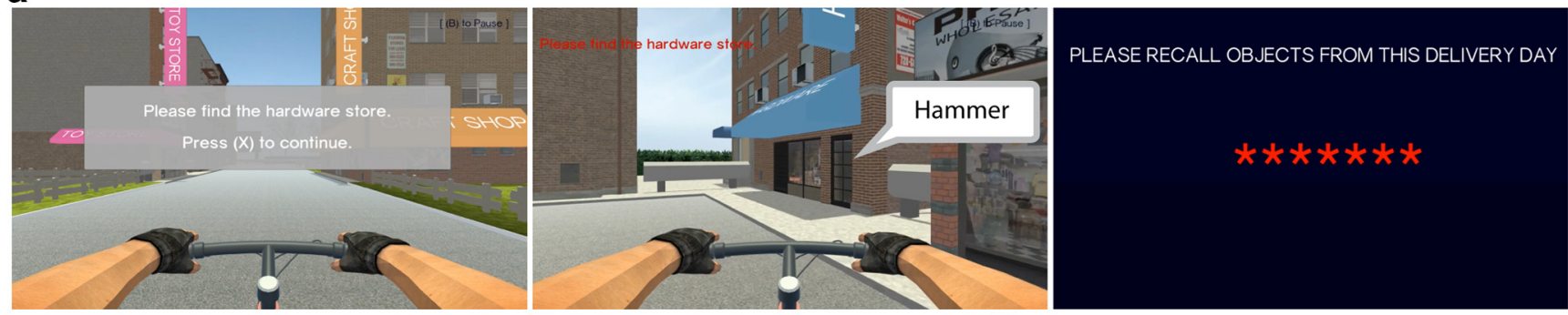

$12 x$

b

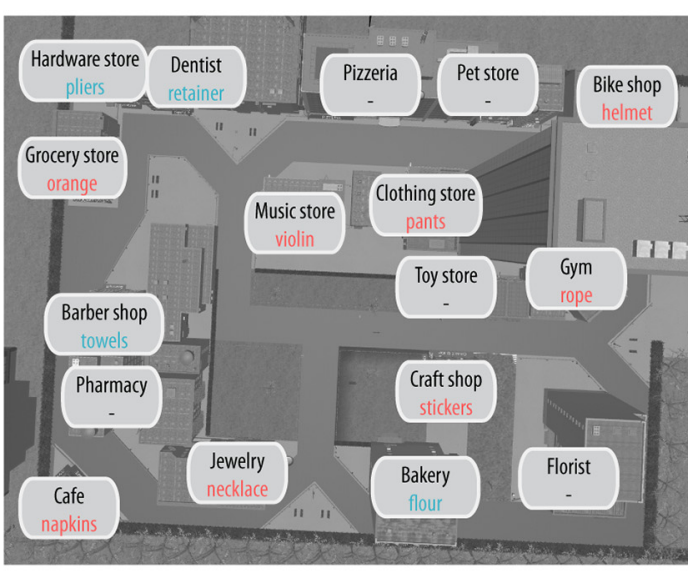

C

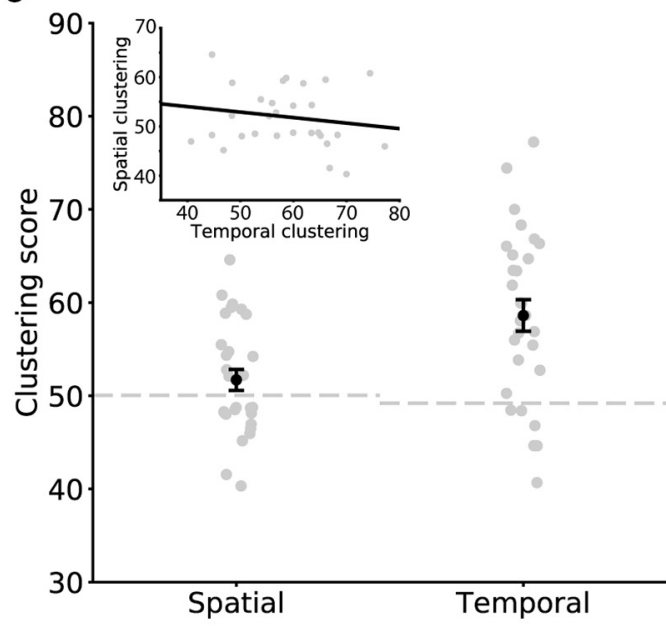

d

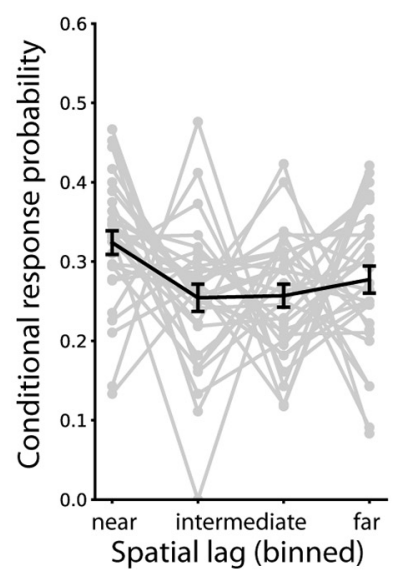

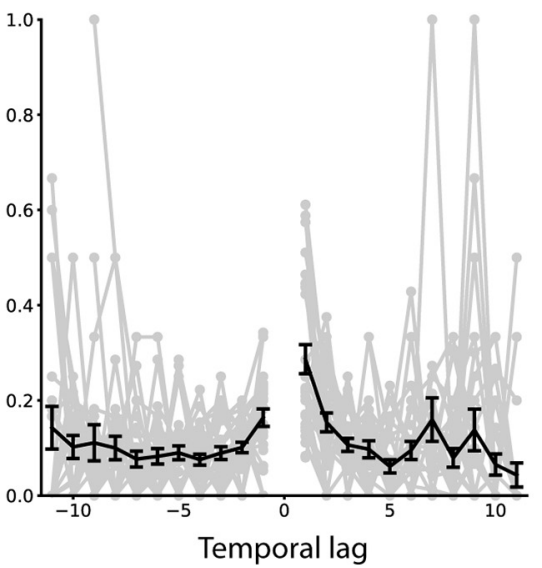

e

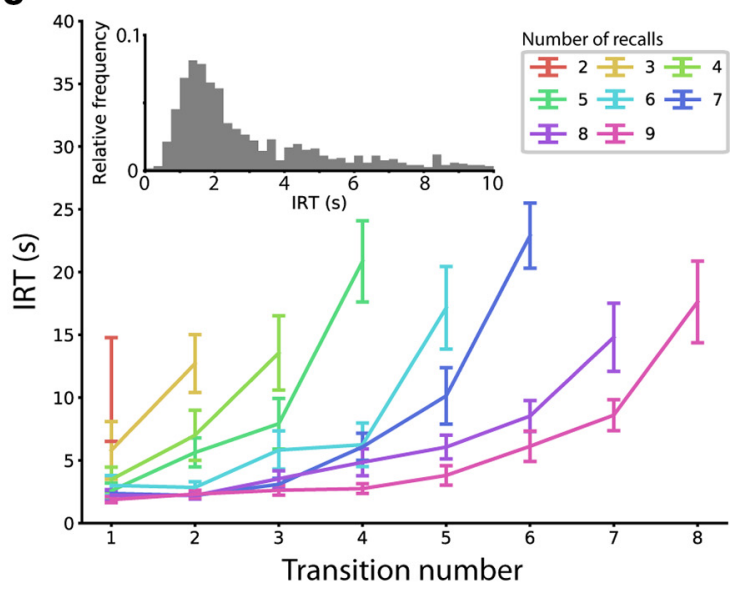

Figure 1. Task design and behavioral clustering. $\boldsymbol{a}$, Hybrid spatial-episodic memory task in which subjects play the role of a courier. On each trial, subjects navigate to 12 different target stores to deliver parcels. Upon arrival, the just-delivered object is revealed. After 12 deliveries, subjects navigate to a final store. Here, the screen goes black and subjects attempt to freely recall all objects they delivered in any order. $\boldsymbol{b}$, Bird's-eye view of the virtual city. Overlaid are store names together with a toy example list. For each recall transition, we calculate a spatial clustering score (SCS) and temporal clustering score (TCS) between 0 and 100 that reflects the spatial or temporal proximity between the recalled words during encoding. $c$, Clustering in recall sequences. Subjects organize their recalls with respect to spatial and temporal context information, as indicated by spatial and temporal clustering scores larger than the permutation average indicated by the dashed lines (higher scores are associated with closer recall transitions; see Materials and Methods). Error bars show SEM across subjects. Spatial and temporal clustering are uncorrelated across subjects (inset). $\boldsymbol{d}$, Conditional response probabilities of transitioning from recall of item i to item j with a given spatial (left) or temporal (right) lag between items i and j. Conditional response probability curves show spatial and temporal contiguity effects in subjects' recall sequences. Error bars show SEM across subjects. $\boldsymbol{e}$, IRTs for correct recalls by transition number shown separately for lists with two to nine correct recalls. The inset shows a histogram of all IRTs shorter than $10 \mathrm{~s}$ pooled across all subjects. Error bars show SEM across subjects.

We used a permutation procedure to assess significance of spatial (temporal) clustering across subjects. To do so, all recalled words on a given trial for a given subject were randomly permuted 2000 times. The distribution of average clustering scores across subjects obtained from these random permutations provides a measure of clustering values observed by chance while controlling for the identity and number of recalled words per trial. The percentage of random clustering scores larger than the observed clustering score constitutes the permutation $p$ value. We used linear mixed-effects models with a random subject intercept to assess the relation between clustering scores and inter-response times (IRTs) and to explore whether clustering scores increased or decreased over the duration of the experiment. In both cases, we compared a model with the predictor of interest (i.e., clustering scores predicting IRT, trial number predicting clustering scores) to an intercept-only model with a likelihood 
ratio test to assess significance. To assess the relation between spatial and temporal clustering across subjects, we computed the correlation between both variables.

Intracranial EEG data acquisition and preprocessing. EEG data were acquired using Ad-Tech Medical Instrument Corporation, PMT Corporation, or Dixi Medical depth electrodes along with a Nihon Kohden, Natus, Compumedics, or IT-med recording system at sampling rates between 400 and $2500 \mathrm{~Hz}$. Coordinates of the radiodense electrode contacts were derived from a postimplant computed tomography or MRI scan and then registered with the preimplant MRI scan in MNI (Montreal Neurological Institute) space using SPM (Wellcome Department of Cognitive Neurology) or Advanced Normalization Tools (https:// github.com/ANTsX/ANTs; Avants et al., 2008). EEG data were analyzed using Python version 2.7 along with the Python Time Series Analysis package (https://github.com/pennmem/ptsa_new). EEG data were aligned with behavioral data via pulses sent from the behavioral testing laptop to the EEG system. We performed our statistical analyses on two distinct time windows: one $750 \mathrm{~ms}$ time window preceding recall $(-750$ to $0 \mathrm{~ms}$ with respect to vocalization onset in the retrieval phase) and one $300 \mathrm{~ms}$ time window during word presentation (100-400 ms with respect to word onset in the encoding phase). We chose the retrieval time window to include retrieval-related neural activity while avoiding overlap with preceding vocalizations (see Fig. $1 e$ for a distribution of IRTs). The encoding time window was used for our encoding-retrieval similarity analysis. Due to our sliding window approach, this window had to be shorter than the retrieval time window. We chose the particular time window as a time period during which subjects were oriented toward encoding the object. To allow for wavelet convolution, we epoched data with a buffer of $2000 \mathrm{~ms}$ on either side (i.e., from -2750 to $2000 \mathrm{~ms}$ with respect to recall onset during retrieval periods, and from -1900 to 2400 $\mathrm{ms}$ with respect to word onset during encoding). Data were re-referenced with a bipolar reference scheme and down-sampled to $400 \mathrm{~Hz}$. A Butterworth filter (order 4; cutoff frequencies $48-52 \mathrm{~Hz}$ for data recorded in Germany and $58-62 \mathrm{~Hz}$ for data recorded in the United States) was used to filter line noise, and subsequently outliers were excluded on an epoch by channel basis. The interquartile range (IQR) was calculated for each channel across all (mean-corrected) encoding or retrieval events within a session. Outliers were identified as samples 5 times the IQR above the 75th percentile or 5 times the IQR below the 25th percentile. Epochs were excluded for a given channel with at least one outlying sample. On average, $2.5 \%$ (minimum, $0 \%$; maximum, $11.0 \%$ ) of epoch-channel pairs were excluded per session and event type. To extract power and phase, the data were convolved with complex Morlet wavelets (five cycles) for 25 log-spaced frequencies between 3 and $100 \mathrm{~Hz}$. After convolution, a buffer was removed at the beginning and end of each epoch, leaving a time window of $100-400 \mathrm{~ms}$ during encoding and -750 to $0 \mathrm{~ms}$ during recall. Data were $z$ scored with respect to the mean and SD across all encoding or recall samples within a session and, depending on the analysis, averaged over time, over frequencies within two frequency bands (theta, $3-8 \mathrm{~Hz}$; high gamma, 70-100 Hz) and over electrodes within two regions of interest (ROIs): HC and PHG, as defined by the Harvard-Oxford Atlas. Subjects' data were included for a given analysis if they contributed at least eight valid trials in at least one ROI (or, where necessary, both ROIs).

Intracranial EEG data statistical analyses. To assess the spectral correlates of successful spatial context retrieval and associated spatial clustering, we used a within-subject linear regression model. The model relates average theta and high gamma power in the $\mathrm{HC}(N=20$ subjects $)$ and PHG $(N=19$ subjects $)$ preceding recall of the first item in a recall transition between two correct recalls to the spatial proximity (i.e., spatial clustering score) associated with that transition. To the extent that retrieved spatial context cues items encoded in close spatial proximity, the spatial proximity of a recall transition should be indicative of spatial context retrieval for the first item in the transition. A $\beta$ value for spatial proximity above zero indicates increased power during retrieval of spatial context, and a value below zero indicates decreased power during retrieval of spatial context. In addition to the spatial clustering score associated with each recall transition, temporal clustering score, serial position, and output position were added as regressors of no interest to account for shared variance with our predictor of interest. The $\beta$ values for spatial clustering scores per region and frequency band for all subjects were analyzed with a linear mixed-effects model, with brain region, frequency, and their interaction as fixed effects and subject as a random intercept effect. Significance of fixed effects was evaluated using likelihood ratio tests between a full model (both main effects or both main effects and their interaction) and a reduced model (one or two main effects) with the effect of interest removed. Differences of average $\beta$ values from zero were assessed with a two-sided one-sample $t$ test.

To test for dynamic spatial context reinstatement, time-frequency spectra ( $25 \mathrm{log}$-spaced frequencies between 3 and $100 \mathrm{~Hz}$ ) during encoding and retrieval were concatenated over all electrodes within either the HC ( $N=20$ subjects $)$ or PHG $(N=20$ subjects $)$. A single vector representing power for all time-frequency-electrode points within a given ROI during encoding was correlated with a corresponding vector for retrieval derived from a sliding time window. The sliding time window equaled the length of the encoding epoch $(300 \mathrm{~ms})$ and was centered on every sample (every $2.5 \mathrm{~ms}$ ) in the retrieval time window $(-750$ to $0 \mathrm{~ms}$ ), located at least half a window size $(150 \mathrm{~ms})$ from the edges of the retrieval time window. For each correct recall event, we calculated the correlation (i.e., neural similarity) with all encoding events on the same trial that did not share the same item. We excluded encoding of the respective item to exclude effects driven by reinstatement of an item as opposed to context information. Each recall event was therefore correlated with 11 encoding events from the same trial. We then used a linear regression model relating (Fisher $z$-transformed) neural similarity at each time point to the spatial proximity (normalized to be between 0 and 1 ) between the locations of the encoded and recalled item. A $\beta$ value $>0$ indicates higher similarity for encoding-recall pairs that share spatial context and, hence, can be interpreted as reactivated spatial context. Again, we included additional regressors of no interest to account for shared variance: temporal proximity (the negative absolute temporal difference between the two encoding time points) and study-test proximity (the temporal difference between encoding and recall time). We used study-test proximity instead of serial and output position because we assumed that similarity would more strongly be affected by the temporal distance between the two events than by the temporal position of each individual event. To quantify the temporal dynamics of reactivated spatial information in the HC and PHG, we used a linear mixed-effects model with brain region, time, and their interaction as fixed effects and subject as a random intercept effect. Significance of fixed effects was evaluated using likelihood ratio tests between a full model (both main effects or both main effects and their interaction) and a reduced model (one or two main effects) with the effect of interest removed.

To assess theta-phase to gamma-amplitude coupling between the HC and PHG $(N=14$ subjects), we calculated the synchronization index (Cohen, 2008). This method determines the modulating (low) frequency by finding a peak in the power spectrum of the high-frequency power envelope. If high-frequency power is time locked to low-frequency phase, high-frequency power should fluctuate at the lower oscillation frequency. In our analysis, we restricted the range of modulating frequencies to the theta band (i.e., 3-8 Hz). After determining the modulating frequency for each channel in the PHG, each recall event, and each extracted high frequency, we extracted the phase of the high-frequency power time series using the Hilbert transform. The synchronization index was then calculated as the consistency across time between the phase of the high-frequency power time series (in the PHG) and the lowfrequency filtered signal (in the HC; Cohen, 2008). We excluded from this calculation 4 of 96 available electrode pairs because they shared a bipolar reference contact, and therefore, phase-amplitude coupling (PAC) could not be clearly attributed to a between-region effect but could also stem from PAC within the HC or PHG. The magnitude of the resulting complex number indicates the extent of synchronization, and the angle indicates the preferred phase offset. We then asked whether PAC (i.e., the magnitude of the synchronization index) between the HC and PHG is a function of spatial clustering. To this end, we used recalllevel linear mixed-effects models to relate (Fisher $z$-transformed) PAC to spatial clustering scores across recall events. We included electrode pair nested in subject as random intercept effects, as well as spatial clustering 

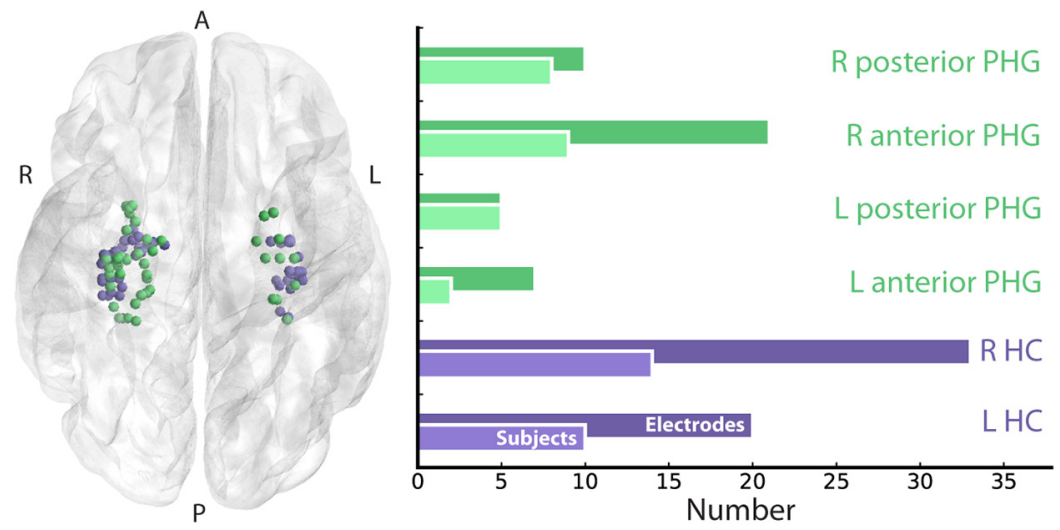

Figure 2. Electrode localization. Electrodes were localized to MTL subregions using the Harvard Oxford Atlas. We collapsed data from the right and left hemispheres as well as anterior and posterior divisions for all analyses. Electrodes colored by ROI (HC, purple; PHG, green) are shown on the left. Electrode and patient breakdowns by subregion are shown on the right.

score and modulated frequency band (high gamma, 70-100 Hz; low gamma, $31-70 \mathrm{~Hz}$; beta, $15-31 \mathrm{~Hz}$ ) as fixed effects. In addition, power in the modulating theta frequency $(3-8 \mathrm{~Hz})$ band was included as a fixed effect to control for the fact that PAC may be confounded by differences in the reliability of phase estimation in time windows of low versus high power. Again, temporal clustering, serial position, and output position were also included as regressors of no primary interest to account for shared variance. Significance of fixed effects was determined using likelihood ratio tests between a full model against a model without the effect in question.

Data availability. Data that can be shared without compromising research participant privacy/consent will be available at http://memory. psych.upenn.edu/Electrophysiological_Data.

\section{Results}

Subjects recalled an average of $49.8 \%$ of words while exhibiting both primacy (serial positions $1-3$ vs $4-9 ; t_{(28)}=2.08, p=0.046$, Cohen's $d=0.39$ ) and recency (serial positions $10-12$ vs $4-9$, $t_{(28)}=5.52, p<0.001$, Cohen's $\left.d=1.03\right)$ effects. They organized their recalls according to both temporal $(p<0.001)$ and spatial ( $p=0.04)$ encoding context, as determined by a permutation test of their spatial and temporal clustering scores (Fig. $1 c, d$; higher scores indicate stronger spatial/temporal organization; see Materials and Methods for details). Thus, subjects tended to successively recall items which were encoded at proximate serial positions or at proximate locations in the virtual environment. These proximate transitions exhibited shorter IRTs than distant transitions, as indicated by a significant effect of spatial $\left(\chi_{(1)}^{2}=\right.$ $4.64, p=0.031)$ and temporal $\left(\chi_{(1)}^{2}=10.89, p=0.001\right)$ clustering scores on IRTs across transitions. This finding aligns with the idea that clustering scores reflect reinstatement of encoding context, which can cue (and thereby facilitate retrieval of) the next retrieved item. We did not find any evidence for a change of spatial $\left(\chi_{(1)}^{2}=0.55, p=0.46\right)$ or temporal $\left(\chi_{(1)}^{2}=0.66, p=0.42\right)$ clustering scores over successive trials. Furthermore, we found no correlation between spatial and temporal clustering scores $\left(r_{(27)}\right.$ $=-0.15, p=0.43$ ) across subjects, suggesting that there is no subject-specific variable, such as associative memory performance or strategy use, that drives clustering behavior.

To identify the spectral signature of spatial context retrieval, we exploited the fact that spatial clustering during recall is indicative of successful retrieval of spatial context information. When spatial context is retrieved, along with an object's identity, it serves as a cue for other objects encoded in a similar spatial context and thereby favors spatially close recall transitions. With this logic in mind, we assessed the role of theta and gamma activity in the $\mathrm{HC}$ and $\mathrm{PHG}$ (see Fig. 2 for electrode locations) for spatial context retrieval using a withinsubject linear regression model. The model relates spectral power preceding vocal recall of each object to the spatial proximity between the encoding locations of that object and the next recalled object (Fig. $3 a$; see Materials and Methods for details). A positive relation (i.e., $\beta$ parameter) would indicate a power increase during retrieval of spatial context, whereas a negative relation would indicate a power decrease.

Figure $3 b$ shows the $\beta$ parameters for spatial proximity across all subjects. We found a significant effect of frequency $\left(\chi_{(1)}^{2}=4.91, p=0.027\right)$, with more positive $\beta$ values for theta than high gamma power. Average $\beta$ values in the $\mathrm{HC}$ and PHG were also significantly larger than zero for theta $\left(t_{(24)}=\right.$ 2.22, $p=0.036$, Cohen's $d=0.44)$ but not gamma $\left(t_{(24)}=1.07\right.$, $p=0.29$, Cohen's $d=0.21)$ power. There was no effect of brain region $\left(\chi_{(1)}^{2}=1.27, p=0.26\right)$ and no interaction $\left(\chi_{(1)}^{2}=0.33, p=\right.$ $0.57)$. The increase in theta power before spatially clustered recalls in the HC and PHG appears prominently in the average raw time-frequency spectra (Fig. $3 c$ ). These results suggest that increased theta power in the HC and PHG accompanies retrieval of spatial information during episodic free recall.

We performed a parallel exploratory analysis to establish whether a similar increase in theta power occurs for transitions that are temporally, rather than spatially, close. Average $\beta$ values relating temporal clustering scores to theta power in the $\mathrm{HC}$ and PHG were not significantly different from zero $\left(t_{(24)}=0.05, p=\right.$ 0.96 , Cohen's $d=0.01)$. Furthermore, in direct comparison, the average increase in theta in the PHG and HC during spatially close transitions was marginally stronger than that during temporally close transitions $\left(t_{(24)}=2.01, p=0.055\right.$, Cohen's $d=$ $0.40)$.

Next, we sought to assess dynamic reinstatement of specific spatial representations in the $\mathrm{HC}$ and $\mathrm{PHG}$. To this end, we used encoding-retrieval similarity analyses with a sliding window approach. Specifically, we correlated a vector representing power during encoding for all electrodes in the HC or PHG, all frequencies, and all time points with a corresponding retrieval vector derived from a sliding time window to track reactivation (i.e., neural similarity) of encoding features leading up to recall (Fig. $4 a$ ). Applying a rationale similar to the one described above, we used the spatial proximity between the locations associated with each encoding and recall event pair to isolate representations of spatial context. The degree to which similarity of encoding and recall events can be explained by the proximity between their associated encoding locations provides an estimate of the amount of reactivation of spatial context information during recall. We estimated this relation in a within-subject approach separately for each time point leading up to recall using linear regression of neural similarity and spatial proximity (see Materials and Methods for details). At each time point, a $\beta$ value $>0$ indicates higher similarity for encoding-recall pairs that share spatial context and, hence, can be interpreted as the instantaneous amount of reactivated spatial information. To avoid confounding this measure with reinstatement of item-level information, we excluded encoding-recall pairs of the same item. 


\section{a Encoding}

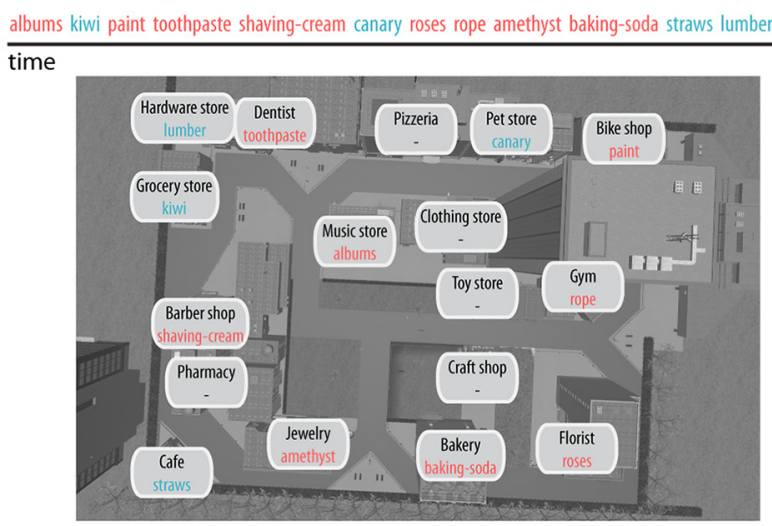

Retrieval
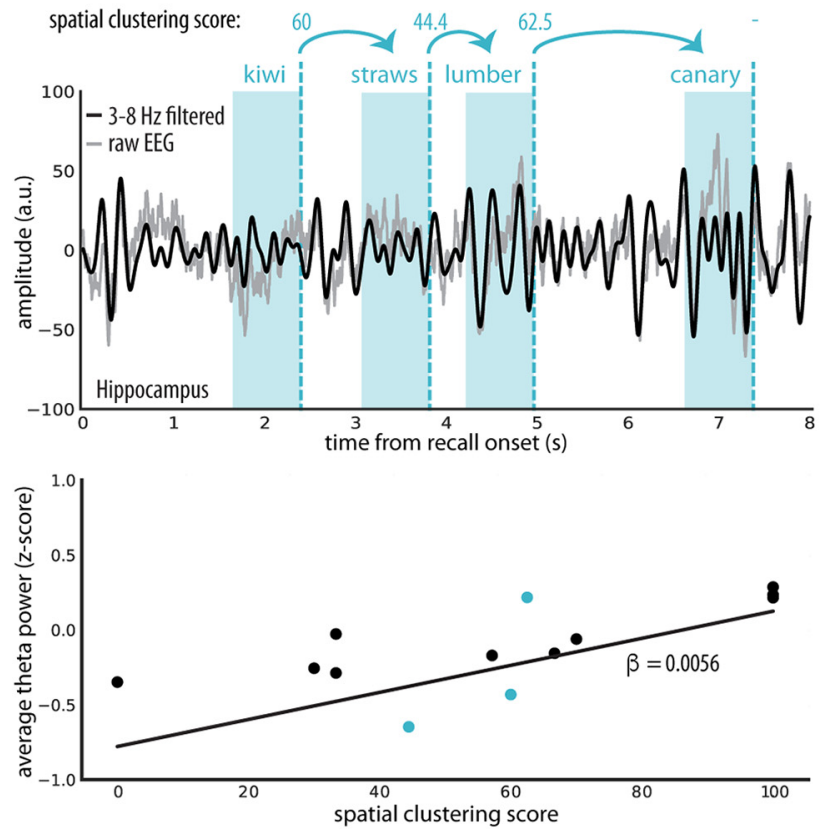

b

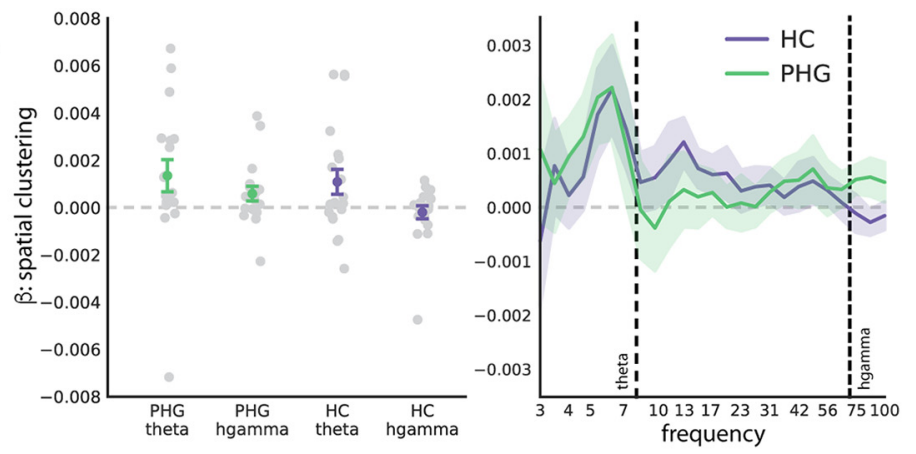

C
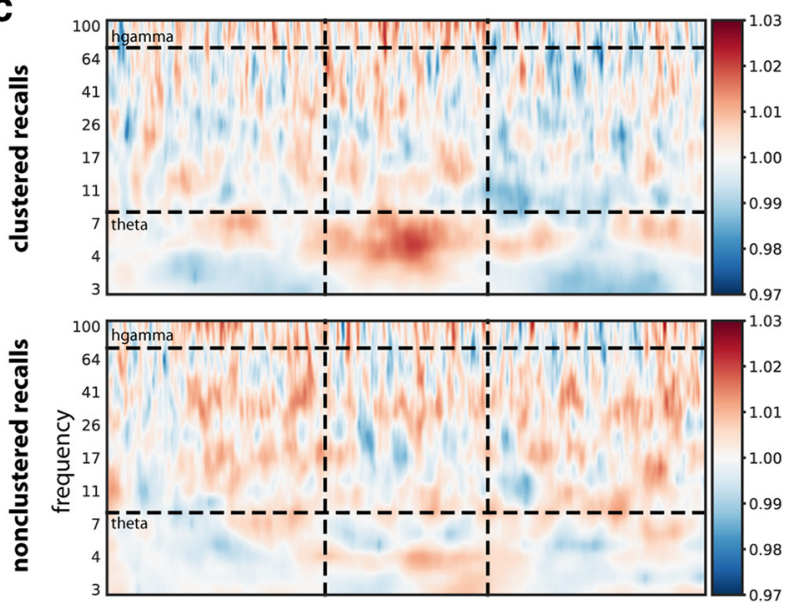

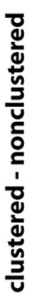

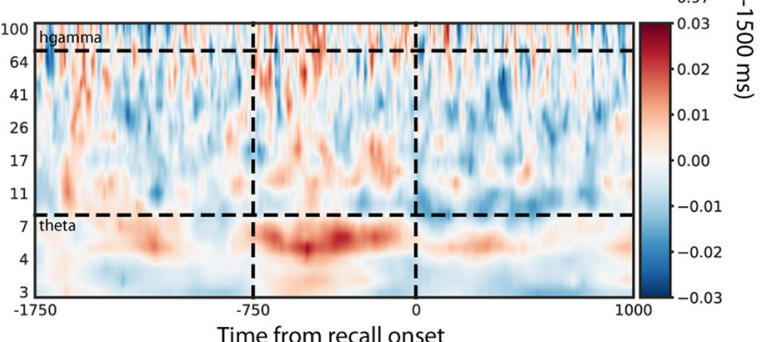

Figure 3. The spectral signature of spatial context retrieval. $\boldsymbol{a}$, Top, Sample encoding trial, in which 12 items were sequentially encoded in different locations of the virtual town. During recall, retrieved context cues items experienced in a similar encoding context. Retrieval of spatial context can therefore be inferred from the spatial proximity (i.e., clustering score) associated with each recall transition. $\boldsymbol{a}$, Middle, An example trace of average hippocampal raw and theta-filtered EEG during recall (power was extracted from -750 to 0 ms before word onset; shaded regions) along with the spatial clustering scores for each transition. $\boldsymbol{a}$, Bottom, We collapsed data from all trials of a given subject and used linear regression to relate power before recall of each word to the spatial clustering score associated with transitioning to the next word. The three transitions illustrated in the hippocampal time series appear as blue dots in the scatter plot. A positive $\beta$ indicates stronger power during retrieval of spatial context. $\boldsymbol{b}$, Left, $\beta$ parameters ( \pm SEM) for all subjects as a function of brain region and frequency band. Theta ( $3-8 \mathrm{~Hz}$ ), but not high gamma (hgamma; $70-100$ $\mathrm{Hz}$ ), power increases in the $\mathrm{HC}$ and PHG during spatial context retrieval. $\boldsymbol{b}$, Right, $\beta$ parameters ( \pm SEM) across the entire frequency spectrum. Strongest increases in power were observed at $\sim 5-7$ Hz. c, Increases in theta power for spatially clustered (spatial clustering score $>70$ ) compared with nonclustered recalls (spatial clustering score $<30$ ) are also visually evident in raw power spectra averaged over the $\mathrm{HC}$ and PHG for all subjects. The average power spectra per subject were baseline corrected with a relative baseline from -1750 to $-1500 \mathrm{~ms}$.

We observed distinct temporal profiles of spatial context reinstatement in the HC and PHG: whereas our index of retrieved spatial information numerically decreased in the HC, it increased in the PHG leading up to recall (Fig. $4 b$ ). To quantify this difference in temporal trend, we used a linear mixed-effects model (see Materials and Methods for details). Using likelihood ratio tests, we observed a main effect of brain region $\left(\chi_{(1)}^{2}=82.04, p<\right.$ 0.001 ) with stronger spatial reactivation in the HC than the PHG, as well as a main effect of time $\left(\chi_{(1)}^{2}=15.73, p<0.001\right)$ with stronger spatial reactivation at early time points. We also observed a region-by-time interaction $\left(\chi_{(1)}^{2}=290.17, p<0.001\right)$, indicating different time courses of reactivation in the $\mathrm{HC}$ and PHG. Reducing the model to a single brain region revealed a negative effect of time in the HC (i.e., spatial information de- creased; $\left.\chi_{(1)}^{2}=176.04, p<0.001\right)$ and a positive effect of time in the PHG (i.e., spatial information increased; $\chi_{(1)}^{2}=299.68, p<$ $0.001)$. Removing all fixed effects in the model revealed a trend for the intercept $(z=1.84, p=0.066)$, which can be interpreted as the overall degree of spatial reactivation regardless of brain region and timing. These results indicate that spatial context reactivation exhibits distinct time courses in the HC and PHG. The finding that our index of retrieved spatial information in the HC appears more strongly earlier in the prevocalization period than in the PHG may suggest information transfer between the HC and PHG.

As a next step, we assessed connectivity between the $\mathrm{HC}$ and PHG as a function of spatial context retrieval. If spatial information is indeed being relayed from the HC to PHG, we should be 
a
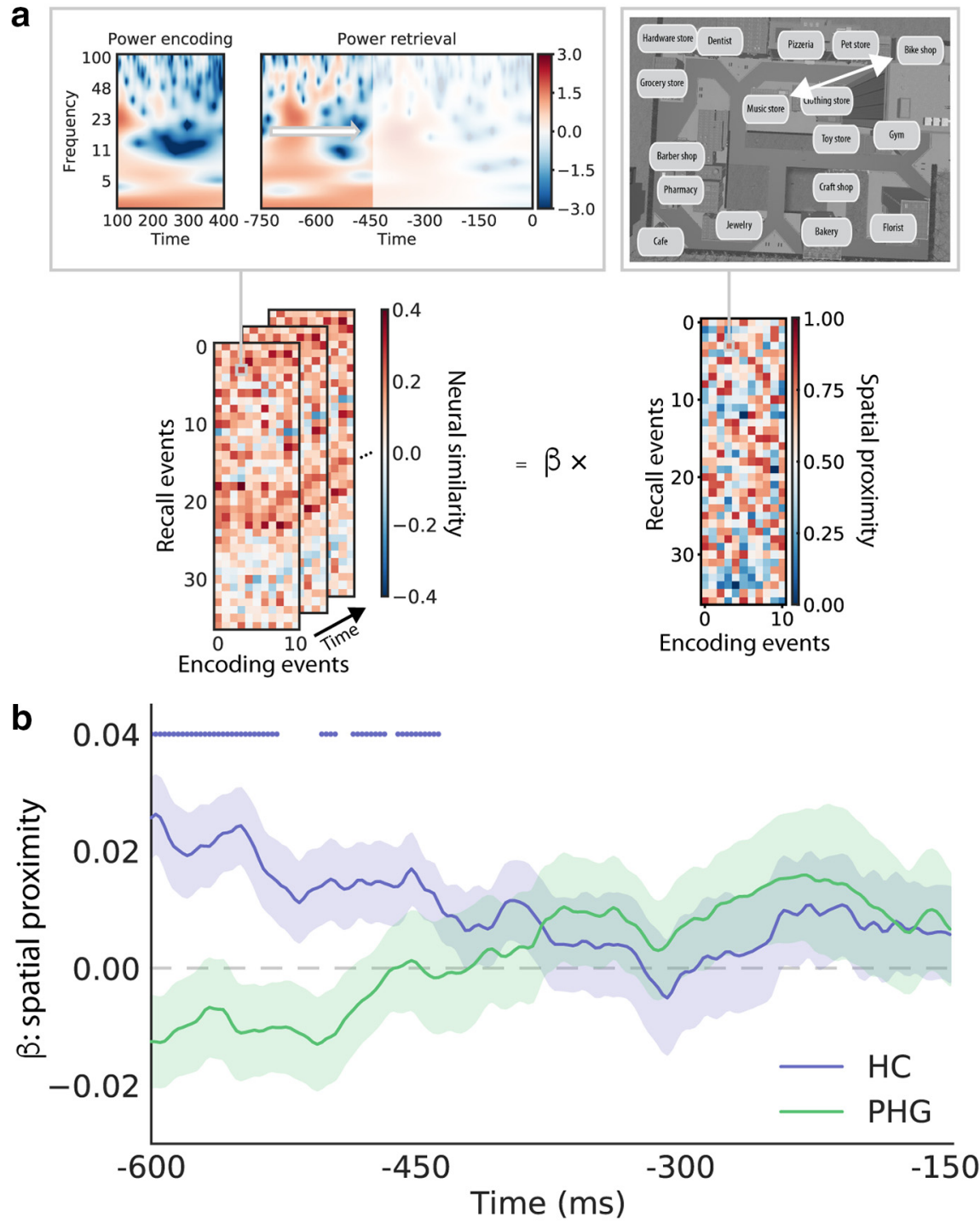

Figure 4. Reinstatement of spatial information leading up to recall. $\boldsymbol{a}$, To obtain an index of retrieved spatial information over time, we first calculated neural encoding-retrieval similarity between each recall event and all encoding events on the same trial. For example, the subject whose data are depicted made 37 correct recalls in total (rows of the similarity matrix). Similarity was calculated between each of these recalls and all encoding events from the same trial (columns of the similarity matrix; 11 encoding events for each trial, excluding encoding of the recalled word itself). Specifically, we calculated similarity between a $300 \mathrm{~ms}$ encoding time window and a sliding $300 \mathrm{~ms}$ retrieval time window centered between -600 and $-150 \mathrm{~ms}$ (i.e., half a window size from the edges of each retrieval epoch). We thereby obtained a measure of neural similarity (i.e., reactivation) over time for each encoding-recall event pair for each subject. Because we were specifically interested in reactivation of spatial information, we then used a within-subject regression model to relate neural encoding-retrieval similarity at each time point to the spatial proximity between the locations associated with the respective encoding and recall events. $\boldsymbol{b}$, The average resulting $\beta$ parameters across subjects ( \pm SEM) are plotted as a function of time, separately for the HC and PHG. Dots above the time series indicate significant time points with $p$ (uncorrected) $<0.05$. A $\beta$ parameter $>0$ indicates higher similarity for encoding-recall pairs that share spatial context and, hence, can be interpreted as reactivated spatial information. Spatial information is represented more strongly in the $\mathrm{HC}$ at the beginning of the epoch. Subsequently, information decreases in the $\mathrm{HC}$ and increases in the PHG leading up to recall.

able to observe connectivity between these brain regions when spatial context is retrieved. Specifically, we explored theta-gamma phase-amplitude coupling between the HC and PHG (i.e., a modulation of parahippocampal gamma amplitude by hippocampal theta phase). To this end, we calculated the synchronization index (Cohen, 2008) between the hippocampal theta phase and the phase of the parahippocampal gamma power envelope for all HC-PHG electrode pairs during recall. We then used a linear mixed-effects model to relate PAC (i.e., the magnitude of the synchronization index) to spatial clustering scores across recall events (see Materials and Methods for details). A positive relation would indicate that retrieval of spatial context is associated with increased thetagamma coupling between the HC and PHG.

Spatial clustering was a significant positive predictor of $\mathrm{PAC}$ in the high gamma band $\left(70-100 \mathrm{~Hz} ; \chi_{(1)}^{2}=5.16, p=0.023\right)$. This means that close spatial recall transitions (i.e., retrieval of spatial context) were associated with an increase in thetagamma coupling between the $\mathrm{HC}$ and PHG. We observed a similar trend in the low gamma band $\left(31-70 \mathrm{~Hz} ; \chi_{(1)}^{2}=3.48\right.$, $p=0.062)$ but no effect in the beta band $\left(15-31 \mathrm{~Hz} ; \chi_{(1)}^{2}=1.10, p=0.29\right)$. When including data for all three frequency bands in the same model, there was a main effect of spatial clustering $\left(\chi_{(1)}^{2}=6.70\right.$, $p=0.010$ ) with higher PAC during spatially clustered recalls and a main effect of frequency $\left(\chi_{(2)}^{2}=1931.02, p<0.001\right)$ with overall higher PAC in lower frequencies, but no interaction of spatial clustering and frequency $\left(\chi_{(2)}^{2}=0.08, p=0.96\right)$. Therefore, it remains to be shown how specific the PAC effect is to the (high) gamma band. Figure 5 shows the $\beta$ estimates for spatial clustering by frequency band, and illustrates the effect of higher PAC for spatially close recall transitions for the high gamma band. We did not observe an effect of temporal clustering in any of the bands (all $p>0.2$ ) or across bands $\left(\chi_{(1)}^{2}=2.59, p=0.11\right)$. We also did not observe a positive spatial clustering effect when reversing direction between the HC and PHG (i.e., parahippocampal theta phase modulating hippocampal highfrequency amplitude during close transitions). The respective $\beta$ estimate was positive but nonsignificant in the high gamma band $\left(\chi_{(1)}^{2}=1.04, p=0.31\right)$ and negative in both other bands. Our results suggest that spatial retrieval is linked to PAC between hippocampal theta and parahippocampal gamma in a broad high-frequency range, implicating PAC in transfer and coding of spatial information in the MTL.

\section{Discussion}

Whereas numerous studies have long implicated temporal context as a powerful organizing principle in episodic retrieval (Kahana, 1996; Healey et al., 2019), the organization of episodic memories by spatial context has only recently been assessed in humans (Gmeindl et al., 2011; Miller et al., 2013a). Our behavioral results demonstrate that when a spatial study context is made available, subjects tend to recall items in succession that were encoded at proximate spatial locations, mirroring the established temporal contiguity effect. We thus find that, similar to temporal context, spatial context is reinstated during episodic retrieval. 

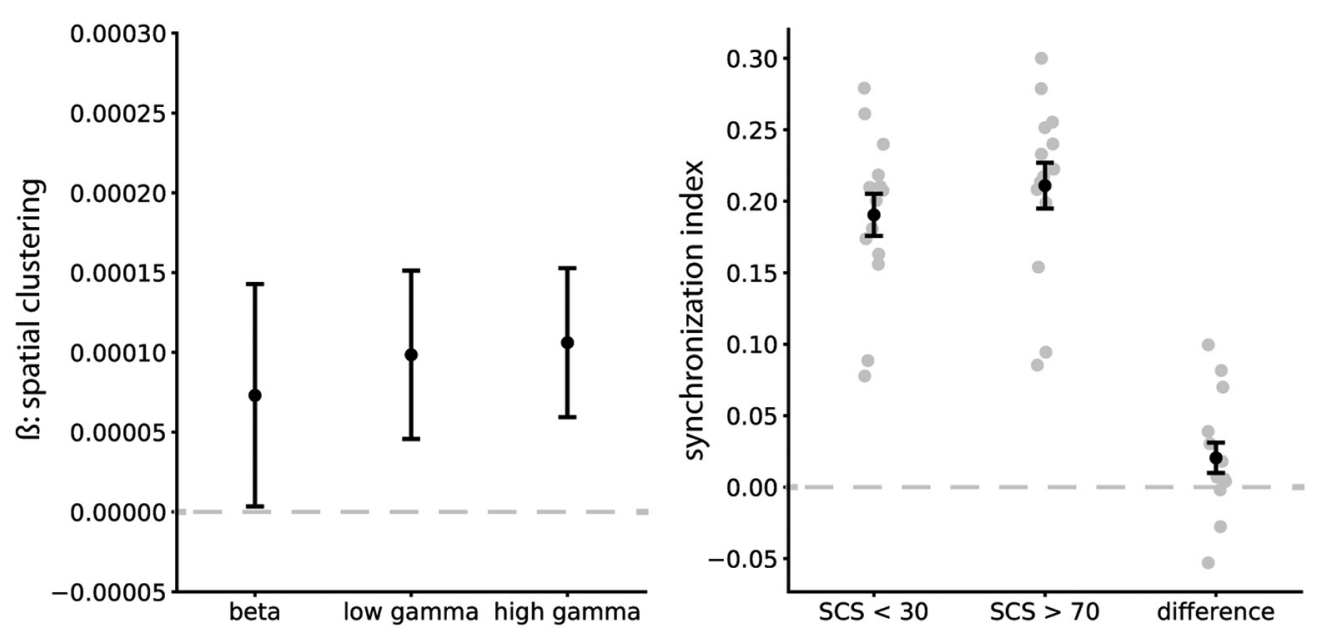

Figure 5. Phase-amplitude coupling between hippocampal theta phase and parahippocampal gamma amplitude. We used a mixed linear model with random subject intercepts to assess the relation between spatial clustering (a measure of spatial context retrieval) and PAC. Left, $\beta$ parameters ( \pm SE) are shown for the high gamma, low gamma, and beta frequency bands. Spatial clustering was a significant positive predictor of PAC between hippocampal theta phase and parahippocampal high gamma amplitude. Right, This relation is illustrated, showing higher PAC for spatially close recall transitions (SCS > 70; i.e., during retrieval of spatial context) than for spatially far transitions (SCS < 30). Error bars show SEM across subjects.

To elucidate the physiological basis of spatial context reinstatement, we related the spatial proximity for each recall transition (a measure of spatial context reinstatement) to spectral power during retrieval. We observed increased medial temporal theta, but not gamma power, during spatial context retrieval. This finding is in line with studies linking theta to recollection (Guderian and Düzel, 2005; Herweg et al., 2016), as well as with studies showing that theta power increases during recall of items that were encoded in semantically structured lists (Weidemann et al., 2019). These findings thereby specifically implicate theta in retrieving an item's encoding context. Our results seem at odds with studies observing a spectral tilt with increased highfrequency power and decreased low-frequency power during successful recall compared with deliberation (Burke et al., 2014; Kragel et al., 2017; Weidemann et al., 2019; Solomon et al., 2019). At a closer look, our results may indicate that a more strongly matched recall contrast (such as recall with vs without context) reveals a neural signature of associative retrieval that is often hidden under a global spectral tilt.

Our findings are of particular interest given the known role of theta oscillations in spatial navigation (Herweg and Kahana, 2018). Theta oscillations increase during movement compared with stillness (Vanderwolf, 1969; Buzsáki et al., 1983; Ekstrom et al., 2005; Aghajan et al., 2017; Bohbot et al., 2017; Bush et al., 2017). In rodents, they organize the spiking of place-responsive cells (O'Keefe and Recce, 1993; Brandon et al., 2011; Koenig et al., 2011). In humans, theta power codes for spatial distances (Vass et al., 2016; Bush et al., 2017) and has been linked to spatial memory: theta power increases during encoding and retrieval of spatial locations (Kaplan et al., 2012; Miller et al., 2018) as well as during successful navigation to a goal (Cornwell et al., 2008). In our task, theta power increased in the absence of any affordances to maintain or recall spatial information. Therefore, our results extend previous findings to suggest that theta oscillations provide a common electrophysiological signature of spatial coding during navigation, explicit spatial memory demands, and incidental episodic retrieval of spatial context information.

But where and when exactly is spatial information retrieved? It has previously been shown that place cells reinstate their activity during recall of items encoded within the place field of a cell (Miller et al., 2013b). The temporal dynamics of reinstatement in different MTL subregions, however, remained unknown. Using encoding-retrieval similarity analysis, we show that spatial information is reinstated with distinct temporal profiles. Whereas spatial information is reinstated early in the HC, information in the PHG increases closer to recall (even though reactivation at individual time points in the PHG is not significantly different from zero). In support of the idea that the HC and PHG interact during retrieval, we also show that theta-phase to gamma-amplitude coupling between the $\mathrm{HC}$ and $\mathrm{PHG}$ increases during retrieval of spatial context.

Our results complement prior studies, which have linked theta-gamma coupling within the $\mathrm{HC}$ as well as theta-gamma coupling between frontal and posterior regions in scalp EEG to successful memory (Mormann et al., 2005; Friese et al., 2013; Lega et al., 2016). They also fit in with prior literature on intraMTL connectivity during retrieval of object-scene pairs: using fMRI, Staresina et al. (2013) observed differential onset latencies in regions of the PHG specialized for objects and scenes, depending on which type of information was presented as the cue and which was to be recalled. Dynamic causal modeling analyses favored a model in which information was routed from the PHG via the HC back to the PHG (Staresina et al., 2013). In a second study, the authors used single-unit recordings from the $\mathrm{HC}$ and EC to show that object reinstatement in the EC is linked to preceding changes in firing rate in the HC (Staresina et al., 2019). Together, the current results and these prior findings suggest that the $\mathrm{HC}$ is the initial locus of retrieval for associations between item and spatial context (or objects and scenes), and that this information is relayed to cortical regions in the PHG. Alternatively, early reactivation in the $\mathrm{HC}$ and later (subthreshold) reactivation in the PHG could be explained by a third source projecting to both regions with distinct time delays. This alternative explanation may be ruled out using direct electrical stimulation to assess the causal relation between early reactivation in the $\mathrm{HC}$ and later reactivation in the $\mathrm{PHG}$.

Future research could also elucidate the nature of neural representations being reinstated in the HC or PHG. Spatially responsive cells have different tuning properties in different MTL subregions. Place cells are mostly found in the CA (cornu ammonis) areas of the $\mathrm{HC}$, whereas grid cells are found in the EC (Ekstrom et al., 2003; Moser et al., 2008; Jacobs et al., 2013; Nadasdy 
et al., 2017). In humans, a different type of cell located in the PHG responds to view of landmarks, regardless of a subject's location (Ekstrom et al., 2003), suggesting that these cells are more sensitive to visual features than abstract location. Therefore, the type of spatial representation that is reinstated may differ, potentially with an abstract to concrete gradient from the $\mathrm{HC}$, over the EC, to the PHG. Whereas our analyses of spatial context reinstatement assumed a linear relation between actual and representational spatial distance (i.e., Fig. 4 shows the linear relation between neural similarity and spatial proximity), it is possible that some regions in the MTL do not represent spatial information on such a linear scale. If the $\mathrm{PHG}$, for instance, primarily represents visual features of scenes, then it might exhibit strong neural similarity at all locations providing a similar view and low similarity otherwise. This would explain why reinstatement in our study was overall stronger in the HC compared with the PHG. More research is needed to understand reactivation of such spatial features that are nonlinearly related to spatial distance.

Having shown that theta power increases during retrieval of spatial context, one may ask whether the role of theta is specific to the representation of spatial information. Alternatively, were theta to play a more general role in context-mediated retrieval, one might predict a positive correlation between theta and retrieval of temporally clustered items, which we did not observe. We do not see the absence of such a correlation, however, as ruling out a general role for theta in contextual retrieval. In our hybrid spatial-episodic memory paradigm, we observe strong primacy and recency effects (as reported above). Primacy effects generally arise from increased attention to, and rehearsal of, early list items (Kahana, 2012). Recency effects result from recencysensitive retrieval processes (Howard and Kahana, 1999; Davelaar et al., 2005). In both of these cases, temporal clustering may not reflect contextual retrieval, but it will appear to the degree that early and late list items tend to be recalled together. Furthermore, in our sample, electrodes were predominantly located in the right MTL, which may be more strongly involved in spatial than in temporal context reinstatement (Kessels et al., 2001; Spiers et al., 2001; Miller et al., 2018).

Our findings indicate that episodic memories are organized by spatial context, resulting in a spatial contiguity effect that parallels the temporal contiguity effect in free recall. We further demonstrate that increased medial temporal theta power accompanies retrieval of spatial context, implicating theta oscillations as a common neurophysiological substrate of spatial coding in navigation and episodic retrieval. Exploring the temporal dynamics of reinstatement in the $\mathrm{HC}$ and $\mathrm{PHG}$, we find that spatial context is initially retrieved in the $\mathrm{HC}$ and emerges later in the PHG. Finally, we demonstrate that hippocampal theta phase modulates parahippocampal gamma amplitude during retrieval of spatial context, suggesting a role for cross-frequency coupling in coding and transmitting retrieved information.

\section{References}

Aghajan ZM, Schuette P, Fields TA, Tran ME, Siddiqui SM, Hasulak NR, Tcheng TK, Eliashiv D, Mankin EA, Stern J, Fried I, Suthana N (2017) Theta oscillations in the human medial temporal lobe during real-world ambulatory movement. Curr Biol 27:3743-3751.e3.

Avants BB, Epstein CL, Grossman M, Gee JC (2008) Symmetric diffeomorphic image registration with cross-correlation: evaluating automated labeling of elderly and neurodegenerative brain. Med Image Anal 12:2641.

Bohbot VD, Copara MS, Gotman J, Ekstrom AD (2017) Low-frequency theta oscillations in the human hippocampus during real-world and virtual navigation. Nat Commun 8:1-7.
Brandon MP, Bogaard AR, Libby CP, Connerney MA, Gupta K, Hasselmo ME (2011) Reduction of theta rhythm dissociates grid cell spatial periodicity from directional tuning. Science 332:595-599.

Burke JF, Sharan AD, Sperling MR, Ramayya AG, Evans JJ, Healey MK, Beck EN, Davis KA, Lucas TH 2nd, Kahana MJ (2014) Theta and highfrequency activity mark spontaneous recall of episodic memories. J Neurosci 34:11355-11365.

Bush D, Bisby JA, Bird CM, Gollwitzer S, Rodionov R, Diehl B, McEvoy AW, Walker MC, Burgess N (2017) Human hippocampal theta power indicates movement onset and distance travelled. Proc Natl Acad Sci U S A 114:12297-12302.

Buzsáki G (2005) Theta rhythm of navigation: link between path integration and landmark navigation, episodic and semantic memory. Hippocampus $15: 827-840$.

Buzsáki G, Leung L-WS, Vanderwolf CH (1983) Cellular bases of hippocampal EEG in the behaving rat. Brain Res Rev 6:139-171.

Cohen MX (2008) Assessing transient cross-frequency coupling in EEG data. J Neurosci Methods 168:494-499.

Cornwell BR, Johnson LL, Holroyd T, Carver FW, Grillon C (2008) Human hippocampal and parahippocampal theta during goal-directed spatial navigation predicts performance on a virtual morris water maze. J Neurosci 28:5983-5990.

Davelaar EJ, Goshen-Gottstein Y, Ashkenazi A, Haarmann HJ, Usher M (2005) The demise of short-term memory revisited: empirical and computational investigations of recency effects. Psychol Rev 112:3-42.

Dragoi G, Buzsáki G (2006) Temporal encoding of place sequences by hippocampal cell assemblies. Neuron 50:145-157.

Ekstrom AD, Kahana MJ, Caplan JB, Fields TA, Isham EA, Newman EL, Fried I (2003) Cellular networks underlying human spatial navigation. Nature 425:184-188.

Ekstrom AD, Caplan JB, Ho E, Shattuck K, Fried I, Kahana MJ (2005) Human hippocampal theta activity during virtual navigation. Hippocampus 15:881-889.

Friese U, Köster M, Hassler U, Martens U, Trujillo-Barreto N, Gruber T (2013) Successful memory encoding is associated with increased crossfrequency coupling between frontal theta and posterior gamma oscillations in human scalp-recorded EEG. Neuroimage 66:642-647.

Gmeindl L, Walsh M, Courtney SM (2011) Binding serial order to representations in working memory: a spatial/verbal dissociation. Mem Cogn 39: $37-46$.

Guderian S, Düzel E (2005) Induced theta oscillations mediate large-scale synchrony with mediotemporal areas during recollection in humans. Hippocampus 15:901-912.

Hafting T, Fyhn M, Molden S, Moser MB, Moser EI (2005) Microstructure of a spatial map in the entorhinal cortex. Nature 436:801-806.

Healey MK, Long NM, Kahana MJ (2019) Contiguity in episodic memory. Psychon Bull Rev 26:699-720.

Herweg NA, Kahana MJ (2018) Spatial representations in the human brain. Front Hum Neurosci 12:297.

Herweg NA, Apitz T, Leicht G, Mulert C, Fuentemilla L, Bunzeck N (2016) Theta-alpha oscillations bind the hippocampus, prefrontal cortex, and striatum during recollection: evidence from simultaneous EEG-fMRI. J Neurosci 36:3579-3587.

Howard MW, Kahana MJ (1999) Contextual variability and serial position effects in free recall. J Exp Psychol Learn Mem Cogn 25:923-941.

Jacobs J, Weidemann CT, Miller JF, Solway A, Burke JF, Wei XX, Suthana N, Sperling MR, Sharan AD, Fried I, Kahana MJ (2013) Direct recordings of grid-like neuronal activity in human spatial navigation. Nat Neurosci 16:1188-1190.

Kahana MJ (1996) Associative retrieval processes in free recall. Mem Cognit 24:103-109.

Kahana MJ (2012) Free recall and memory search. In: Foundations of human memory, pp 185-223. New York: Oxford UP.

Kaplan R, Doeller CF, Barnes GR, Litvak V, Düzel E, Bandettini PA, Burgess N (2012) Movement-related theta rhythm in humans: coordinating self-directed hippocampal learning. PLoS Biol 10:e1001267.

Kessels RP, de Haan EH, Kappelle LJ, Postma A (2001) Varieties of human spatial memory: a meta-analysis on the effects of hippocampal lesions. Brain Res Rev 35:295-303.

Koenig J, Linder AN, Leutgeb JK, Leutgeb S (2011) The spatial periodicity of grid cells is not sustained during reduced theta oscillations. Science 332 592-595. 
Kragel JE, Ezzyat Y, Sperling MR, Gorniak R, Worrell GA, Berry BM, Inman C, Lin JJ, Davis KA, Das SR, Stein JM, Jobst BC, Zaghloul KA, Sheth SA, Rizzuto DS, Kahana MJ (2017) Similar patterns of neural activity predict memory function during encoding and retrieval. Neuroimage 155: $60-71$.

Lega BC, Jacobs J, Kahana M (2012) Human hippocampal theta oscillations and the formation of episodic memories. Hippocampus 22:748-761.

Lega B, Burke J, Jacobs J, Kahana MJ (2016) Slow-theta-to-gamma phaseamplitude coupling in human hippocampus supports the formation of new episodic memories. Cereb Cortex 26:268-278.

Lisman JE, Jensen O (2013) The theta-gamma neural code. Neuron 77:10021016.

Miller JF, Lazarus EM, Polyn SM, Kahana MJ (2013a) Spatial clustering during memory search. J Exp Psychol Learn Mem Cogn 39:773-781.

Miller JF, Neufang M, Solway A, Brandt A, Trippel M, Mader I, Hefft S, Merkow MB, Polyn SM, Jacobs J, Kahana MJ, Schulze-bonhage A (2013b) Neural activity in human hippocampal formation reveals the spatial context of retrieved memories. Science 365:1111-1114.

Miller J, Watrous AJ, Tsitsiklis M, Lee SA, Sheth SA, Schevon CA, Smith EH, Sperling MR, Sharan A, Asadi-Pooya AA, Worrell GA, Meisenhelter S, Inman CS, Davis KA, Lega B, Wanda PA, Das SR, Stein JM, Gorniak R, Jacobs J (2018) Lateralized hippocampal oscillations underlie distinct aspects of human spatial memory and navigation. Nat Commun 9:2423.

Mormann F, Fell J, Axmacher N, Weber B, Lehnertz K, Elger CE, Fernández G (2005) Phase/amplitude reset and theta-gamma interaction in the human medial temporal lobe during a continuous word recognition memory task. Hippocampus 15:890-900.

Moser EI, Kropff E, Moser MB (2008) Place cells, grid cells, and the brain's spatial representation system. Annu Rev Neurosci 31:69-89.

Nadasdy Z, Nguyen TP, Török Á, Shen JY, Briggs DE, Modur PN, Buchanan RJ (2017) Context-dependent spatially periodic activity in the human entorhinal cortex. Proc Natl Acad Sci U S A 114:E3516-E3525.

O'Keefe J (1979) A review of the hippocampal place cells. Prog Neurobiol 13:419-439.

O'Keefe J, Recce ML (1993) Phase relationship between hippocampal place units and the EEG theta rhythm. Hippocampus 3:317-330.

Senior TJ, Huxter JR, Allen K, O’Neill J, Csicsvari J (2008) Gamma oscillatory firing reveals distinct populations of pyramidal cells in the CA1 region of the hippocampus. J Neurosci 28:2274-2286.

Skaggs WE, McNaughton BL, Wilson MA, Barnes CA (1996) Theta phase precession in hippocampal neuronal populations and the compression of temporal sequences. Hippocampus 6:149-172.

Solomon EA, Kragel JE, Sperling MR, Sharan A, Worrell G, Kucewicz M, Inman CS, Lega B, Davis KA, Stein JM, Jobst BC, Zaghloul KA, Sheth SA, Rizzuto DS, Kahana MJ (2017) Widespread theta synchrony and highfrequency desynchronization underlies enhanced cognition. Nat Commun 8:1704.

Solomon EA, Stein JM, Das S, Gorniak R, Sperling MR, Worrell G, Inman CS, Tan RJ, Jobst BC, Rizzuto DS, Kahana MJ (2019) Dynamic theta networks in the human medial temporal lobe support episodic memory. Current Biology 29:1100-1111.

Solway A, Miller JF, Kahana MJ (2013) PandaEPL: a library for programming spatial navigation experiments. Behav Res Methods 45:1293-1312.

Spiers HJ, Burgess N, Maguire EA, Baxendale SA, Hartley T, Thompson PJ, O'Keefe J (2001) Unilateral temporal lobectomy patients show lateralized topographical and episodic memory deficits in a virtual town. Brain 124:2476-2489.

Squire LR, Zola-Morgan S (1991) The medial temporal lobe memory system. Science 253:1380-1386.

Staresina BP, Cooper E, Henson RN (2013) Reversible information flow across the medial temporal lobe: the hippocampus links cortical modules during memory retrieval. J Neurosci 33:14184-14192.

Staresina BP, Reber TP, Niediek J, Boström J, Elger CE, Mormann F (2019) Recollection in the human hippocampal-entorhinal cell circuitry. Nat Commun 10:1503.

Vanderwolf $\mathrm{CH}$ (1969) Hippocampal electrical activity and voluntary movement in the rat. Electroencephalogr Clin Neurophysiol 26:407-418.

Vass LK, Copara MS, Seyal M, Shahlaie K, Farias ST, Shen PY, Ekstrom AD (2016) Oscillations go the distance: low-frequency human hippocampal oscillations code spatial distance in the absence of sensory cues during teleportation. Neuron 89:1180-1186.

Watrous AJ, Tandon N, Conner CR, Pieters T, Ekstrom AD (2013) Frequency-specific network connectivity increases underlie accurate spatiotemporal memory retrieval. Nat Neurosci 16:349-356.

Weidemann CT, Kragel JE, Lega BC, Worrell GA, Sperling MR, Sharan AD, Jobst BC, Khadjevand F, Davis KA, Wanda PA, Kadel A, Rizzuto DS, Kahana MJ (2019) Neural activity reveals interactions between episodic and semantic memory systems during retrieval. J Exp Psychol Gen 148: $1-12$. 